\title{
Renormalization algorithm with graph enhancement
}

\author{
R. Hübener, C. Kruszynska, L. Hartmann, and W. Dür \\ Institut für Theoretische Physik, Universität Innsbruck, Technikerstraße 25, A-6020 Innsbruck, Austria \\ Institut für Quantenoptik und Quanteninformation der Österreichischen Akademie der Wissenschaften, Innsbruck, Austria
}

F. Verstraete

Fakultät für Physik, Universität Wien, Boltzmanngasse 5, A-1090 Wien, Austria

\author{
J. Eisert and M.B. Plenio \\ Institute for Mathematical Science, Imperial College London, London SW7 2PG, UK and \\ Blackett Laboratory, Imperial College London, London SW7 2BW, UK
}

(Dated: November 1, 2018)

\begin{abstract}
We introduce a class of variational states to describe quantum many-body systems. This class generalizes matrix product states which underly the density-matrix renormalization group approach by combining them with weighted graph states. States within this class may (i) possess arbitrarily long-ranged two-point correlations, (ii) exhibit an arbitrary degree of block entanglement entropy up to a volume law, (iii) may be taken translationally invariant, while at the same time (iv) local properties and two-point correlations can be computed efficiently. This new variational class of states can be thought of as being prepared from matrix product states, followed by commuting unitaries on arbitrary constituents, hence truly generalizing both matrix product and weighted graph states. We use this class of states to formulate a renormalization algorithm with graph enhancement (RAGE) and present numerical examples demonstrating that improvements over density-matrix renormalization group simulations can be achieved in the simulation of ground states and quantum algorithms. Further generalizations, e.g., to higher spatial dimensions, are outlined.

PACS numbers: 03.67.Hk,03.65.Ud
\end{abstract}

Strongly correlated quantum systems give rise to a number of intriguing phenomena in condensed matter systems such as the existence of rare-earth magnetic insulators or hightemperature superconductors. The classical description of such quantum many-body systems is difficult, as entanglement and interactions cannot be neglected. A full solution of the underlying microscopic model is unfeasible due to the exponential growth of the dimension of the Hilbert space with the number of constituent particles. In turn, numerical variational approaches, like the density-matrix renormalization group (DMRG) technique [1, 2], make use of an important observation. Typically, ground or thermal states do not occupy the exponentially large Hilbert space, but a much smaller subspace. DMRG can indeed be seen as a variation over the polynomially sized set of matrix product states (MPS) [3, 4, 5, 6], approximating the true ground state iteratively. This approach is expected to work particularly well in onedimensional gapped systems, in which correlation functions decay exponentially and the entanglement entropy saturates at larger block sizes, satisfying an "area law" [7].

Any such variational method, however, has its limitations. For example, in a critical one-dimensional system, the MPS description is no longer economical, with other variational sets potentially being more appropriate. When it comes to time evolution, area laws may be replaced by volume laws [9], and a DMRG picture can become very expensive. Projected entangled pair states (PEPS) form higher-dimensional analogues of matrix product states MPS [12]. This approach is very promising but still in development. For critical systems, multi-scale entanglement renormalization or contractor renormalization [10, 11] are promising candidates also in two dimensions, but are not easily reconcilable with translational invariance. Weighted graph states (WGS) [13, 14, 15] are a family of states that can embody long-range correlations in any spatial dimensions, but do not seem to grasp short-range properties as well as MPS do [16].

With these observations in mind, one of the key questions seems to be the following. How far can one go with the efficient classical description of quantum many-body systems? Can MPS for example be generalized to a larger class of states encompassing some of the above approaches while retaining all of their convenient features? Can one have additional longrange correlations while still being able to efficiently compute local properties and correlation functions? In particular, given the complementary strengths of the MPS and the WGS approach it is natural to attempt a unification of the two approaches. This work shows that indeed the two pictures can be combined to form a new enlarged variational set, while retaining all of the desirable structural elements of its ancestors. We first define the set, discuss variations, sketch generalizations and finally demonstrate applicability and performance as well as limitations in ground state approximations and simulations of quantum algorithms.

Renormalization algorithm with graph enhancement. - We start from MPS of a quantum chain of length $N$, consisting of $d$-level systems, as used in DMRG [3, [4, 5, 6]

$$
|\psi(A)\rangle:=\sum_{s_{1}, \ldots, s_{N}=0}^{d-1} \operatorname{tr}\left[A_{s_{1}}^{(1)} \ldots A_{s_{N}}^{(N)}\right]\left|s_{1}, \ldots, s_{N}\right\rangle
$$


where the $A_{s_{n}}^{(n)}$ are complex $D \times D$ matrices. For open boundary conditions, the left- and rightmost matrices can be taken to be vectors. For simplicity of notation, but in a way that can be trivially generalized, we now fix $d=2$. MPS have correlation functions $\left\langle Z^{(j)} Z^{(j+k)}\right\rangle-\left\langle Z^{(j)}\right\rangle\left\langle Z^{(j+k)}\right\rangle$ exponentially decaying in $k$ and satisfy an area law [7] by construction [19]. An area law in 1D implies that any Renyi entropy $S_{\alpha}$ of the reduced state of a block of $L$ contiguous spins will eventually saturate $\left(S_{\alpha}\left(\rho_{L}\right)=O(1)\right)$; many ground states possess this property and hence a good and economical MPS approximation of them is possible [8].

Now we go beyond this picture and apply to the MPS any set of commuting unitaries between any two constituents, irrespective of the distance. More specifically, we consider the adjacency matrix $\Phi$ of a weighted simple graph with $\Phi_{k, l} \in[0,2 \pi)$ and apply without loss of generality the corresponding phase gates $U\left(\Phi_{k, l}\right):=|0,0\rangle\langle 0,0|+| 0,1\rangle\langle 0,1|+$ $|1,0\rangle\langle 1,0|+| 1,1\rangle\langle 1,1| e^{i \Phi_{k, l}}$ between the particles $k, l$ in the chain. Finally, we apply local rotations $V_{j} \in U(2)$, to arrive at the variational class of states defined by

$$
\begin{aligned}
|\psi(A, \Phi, V)\rangle & :=\prod_{j=1}^{N} V_{j}^{(j)} \prod_{k, l} U^{(k, l)}\left(\Phi_{k, l}\right) \\
& \times \sum_{s_{1}, \ldots, s_{N}} \operatorname{tr}\left[A_{s_{1}}^{(1)} \ldots A_{s_{N}}^{(N)}\right]\left|s_{1}, \ldots, s_{N}\right\rangle,
\end{aligned}
$$

which then forms the basis of the renormalization group algorithm with graph enhancement (RAGE). The above set clearly embodies a large variational class. By definition, for $\Phi=0$ and $V_{j}=\mathbb{1}$, it includes the MPS. It also includes superpositions of WGS as first considered in Ref. [15],

$$
\begin{aligned}
|\varphi\rangle & =\sum_{m} \alpha_{m} \prod_{j=1}^{N} V_{j}^{(j)} \sum_{s_{1}, \ldots, s_{N}=0}^{1} e^{-i \mathbf{s}^{T} \Phi \mathbf{s}+\mathbf{d}_{m}^{T} \mathbf{s}}\left|s_{1}, \ldots, s_{N}\right\rangle \\
& =\prod_{j=1}^{N} V_{j}^{(j)} \prod_{k, l} U^{(k, l)}\left(\Phi_{k, l}\right) \sum_{m} \alpha_{m}\left|\eta_{m, 1}\right\rangle \otimes \ldots \otimes\left|\eta_{m, N}\right\rangle
\end{aligned}
$$

where $\mathbf{d}_{m}=\left(d_{m, 1}, \ldots, d_{m, N}\right), \mathbf{s}=\left(s_{1}, \ldots, s_{N}\right),\left|\eta_{m, n}\right\rangle:=$ $|0\rangle+e^{d_{m, n}}|1\rangle$ and $U\left(\Phi_{m, n}\right)$ are defined as above, and which can be shown to be of the form of Eq. (2). For simplicity, and w.l.o.g, we will often set $V_{j}=\mathbb{1}$ subsequently.

Main properties of RAGE states. - To start with, RAGE states have a polynomially sized description, where the MPS and the WGS part are fully determined by $O\left(N D^{2}\right)$ and $O\left(N^{2}\right)$ real parameters respectively. Furthermore

(i) Volume law for the entanglement entropy: By having a collection of maximally entangled qubit pairs across a boundary, the von-Neumann entropy of a block of length $L$ can be taken to scale as $S\left(\rho_{L}\right)=O(L)$. Encompassing graph states, our class can hence maximize the entanglement entropy.

(ii) Translational invariance: Whenever the MPS part is translationally invariant, $\Phi$ is a cyclic matrix, and $V_{j}$ is the same for all $j$, the whole state $|\varphi\rangle$ is manifestly translationally invariant. There exist other translational invariant states that do not have this simple form. The key feature, though, is that unlike for multiscale entanglement renormalization [10], there exists this natural subset of states for which translational invariance is guaranteed to be exactly fulfilled, while at the same time a volume law for block-wise entanglement is possible [13, 14].

(iii) Completeness: As MPS already form a complete set in Hilbert space (if one allows $D$ to scale as $O\left(2^{N}\right)$, one can represent any pure state in $\left.\left(\mathbb{C}^{2}\right)^{\otimes N}\right)$ and this remains true for the RAGE set.

Efficient computation of local properties and correlation functions. - The previous properties are all very natural and desirable, and especially (i) cannot be achieved efficiently with MPS alone. However, as will be shown, this does not prevent us from computing local properties and correlation functions efficiently - which is the key feature of this set.

To compute expectation values of observables with small support we use the relevant reduced density matrix $\rho_{\mathcal{S}}$, which may be computed efficiently with an effort of $O\left(N D^{5}\right)$ in the total size $N$ of the system $\mathcal{S} \subset\{1, \ldots, N\}$. Controlled phase gates acting exclusively on qubits that are traced out make no contribution, while those that act on the spins in $\mathcal{S}=\left\{m_{1}, \ldots, m_{|\mathcal{S}|}\right\}$ amount to a redefinition of the observables that are local to $\mathcal{S}$. Therefore we redefine $\Phi$ such that the above simplifications are enforced, $\omega_{k, l}=\Phi_{k, l}$ if $k \in \mathcal{S}, l \in$ $\overline{\mathcal{S}}$ or $k \in \overline{\mathcal{S}}, l \in \mathcal{S}$, where $\overline{\mathcal{S}}=\{1, \ldots, N\} \backslash \mathcal{S}$, and $\omega_{k, l}=0$ otherwise. We also define $E_{k, l}^{(j)}:=A_{k}^{(j)} \otimes\left(A_{l}^{(j)}\right)^{*}$, where * denotes complex conjugation. The reduced density matrix $\rho_{\mathcal{S}}$ (up to phase gates in $\mathcal{S}$ ) is then found to be

$$
\begin{aligned}
\rho_{\mathcal{S}}= & \sum_{\substack{s_{1}, \ldots, s_{N}=0 \\
r_{1}, \ldots, r_{N}=0}}^{1} \operatorname{tr}\left[E_{s_{1}, r_{1}}^{(1)} \ldots E_{s_{N}, r_{N}}^{(N)}\right] \operatorname{tr}_{\overline{\mathcal{S}}}\left[\left(\prod_{k, l} U^{(k, l)}\left(\omega_{k, l}\right)\right)\right. \\
& \left.\times\left|s_{1}, \ldots, s_{N}\right\rangle\left\langle r_{1}, \ldots, r_{N}\right|\left(\prod_{k, l} U^{(k, l) \dagger}\left(\omega_{k, l}\right)\right)\right] \\
= & \sum_{\substack{s_{1}, \ldots, s_{N}=0 \\
r_{1}, \ldots, r_{N}=0}}^{1} \operatorname{tr}\left[E_{s_{1}, r_{1}}^{(1)} \ldots E_{s_{N}, r_{N}}^{(N)}\right]\left|s_{m_{1}}, \ldots, s_{m_{|\mathcal{S}|} \mid}\right\rangle \\
& \times\left\langle r_{m_{1}}, \ldots, r_{m_{|\mathcal{S}|} \mid}\right| \prod_{k \in \mathcal{S}, l \in \overline{\mathcal{S}}} e^{i \omega_{k, l}\left(\delta_{s_{k}, 1}-\delta_{r_{k}, 1}\right) \delta_{s_{l}, 1}}
\end{aligned}
$$

The key of the above argument is that the effect of the phases is a mere modification of the transfer operators of the MPS by a phase factor, the phase depending on the matrix element in question. Thus, the evaluation of expectation values is performed using (products of) transfer operators associated with the single sites. The reduced state can then be written as

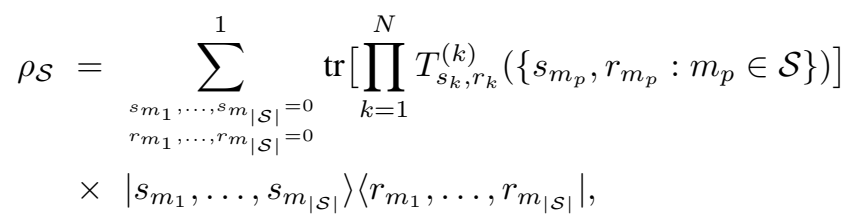

where now $T_{s_{k}, r_{k}}^{(k)}\left(\left\{s_{m_{p}}, r_{m_{p}}\right\}\right):=E_{s_{k}, r_{k}}^{(k)}$ if $k \in \mathcal{S}$, which 
are the unmodified transfer operators of MPS, and

$$
\begin{aligned}
T_{s_{k}, r_{k}}^{(k)}\left(\left\{s_{m_{p}}, r_{m_{p}}\right\}\right) & :=\sum_{l=0}^{1} B_{l}^{(k)}\left(\left\{s_{m_{p}}\right\}, s_{k}\right) \\
& \otimes\left(B_{l}^{(k)}\left(\left\{r_{m_{p}}\right\}, r_{k}\right)\right)^{*}
\end{aligned}
$$

if $k \in \overline{\mathcal{S}}$, which are the transfer operators modified by phases, $B_{l}^{(k)}\left(\left\{s_{m_{p}}\right\}, s_{k}\right):=A_{l}^{(k)} \prod_{m_{p} \in \mathcal{S}} e^{i \omega_{m_{p}, k} \delta_{s_{m_{p}}, 1} \delta_{s_{k}, 1}}$. Grouped in this way, the reduced density operator can indeed be evaluated efficiently. In fact, for each $\left\{r_{m_{p}}, s_{m_{p}}\right\}$ the effort to compute the entry of the reduced state is merely $O\left(N D^{5}\right)$, as one has to multiply $N$ transfer matrices of dimension $D^{2} \times D^{2}$, just as in the case of MPS. This procedure is inefficient in $|\mathcal{S}|$, with an exponential scaling effort. However, any Hamiltonian with two-body (possibly long-ranged) interactions can be treated efficiently term by term.

Efficient updates. - Besides procedures for the efficient computation of reduced density matrices, and therefore expectation values, we need a variational principle to improve the trial states. We will focus on local variational approaches to approximate ground states by minimizing the energy

$$
E:=\frac{\langle\psi(A, \Phi, V)|H| \psi(A, \Phi, V)\rangle}{\langle\psi(A, \Phi, V) \mid \psi(A, \Phi, V)\rangle}
$$

on the approximation of time evolution and on the simulation of quantum circuits. The search for ground states is well known to be related to imaginary-time evolution.

Static updates. - The MPS part can be updated as in variants of DMRG [6]. The expression $\langle\psi(A, \Phi, V)|H| \psi(A, \Phi, V)\rangle$ is (as in MPS) a quadratic form in each of the entries of the matrices $A_{0}^{(k)}, A_{1}^{(k)}$ for each site $k=1, \ldots, N$. An optimal local update can therefore be found by means of solving generalized eigenvalue problems with an effort of $O\left(D^{3}\right)$. Local rotations can be incorporated by parametrizing single qubit rotations on spin $k$ with real parameters $x_{k} \in \mathbb{R}^{4}$ as $V_{k}=\sum_{j=1}^{4} x_{k, j} M_{j}$ with $M=\left(\mathbb{1}, \sigma_{z}, \sigma_{y}, \sigma_{x}\right)$ being the vector of the Pauli matrices including the identity. Again, the local variation of $x_{k}$ in (4) is a generalized eigenvalue problem in $x_{k}$ for each site $k=1, \ldots, N$. To optimize the phases of the WGS, one may first define the new Hamiltonian $H_{V}:=\left(\prod_{j=1}^{N} V_{j}^{(j) \dagger}\right) H\left(\prod_{k=1}^{N} V_{k}^{(k)}\right)$. The optimal phase gates between any pair of spins $j, k \in\{1, \ldots, N\}$ can be computed efficiently as the procedure amounts to a quadratic function of a single variable $z=e^{i \Phi_{j, k}}$. To summarize, an update of $|\psi(A, \Phi, V)\rangle$ to minimize (4) corresponds to a sweeping over such local variations, each of which is efficiently possible, with an effort of $O\left(M N D^{3}\right)$ for $M$ sweeps. An element that is not present for MPS alone is that one can make a choice whether one adapts an MPS part or the adjacency matrix for an identical change in the physical state. In practice, we have supplemented this procedure with a gradient-based global optimization, making use of the fact that the gradient can be explicitly computed.
We have applied the RAGE-method to proof-of-principle 1D and 2D models, where the adjacency matrix is allowed to connect any constituents in the lattice. Fig. 1 a) shows results for the 2D Ising model with transversal magnetic field, $H=\sum_{\langle a, b\rangle} \sigma_{z}^{(a)} \sigma_{z}^{(b)}+B \sum_{a} \sigma_{x}^{(a)}$, comparing the achievable accuracy of MPS (using a one-dimensional path in the 2D lattice) and the RAGE-method for a fixed total number of free parameters. The RAGE-method gives a significantly better accuracy regarding ground state energy and two-point correlations, already for a very small number of parameters. For other models, we see a similar improvement of RAGE over MPS, although in some cases (e.g., for a 2D Heisenberg model) the overall accuracy is still not very satisfactory, possibly related to local minima encountered in the procedure. This new class of states does allow for new features like longrange correlations and a violation of an area law, but in turn, breaks the local $S U(2)$ gauge invariance. It is also clear from the simulations that the limitation of the underlying 1D structure of the MPS cannot always be fully overcome by the graph enhancement. The full potential in numerical performance in identifying ground states is yet to be explored. There exist, however, a number of interesting parent Hamiltonians where the RAGE method should be particularly well suited, e.g., perturbations of models which have a WGS as an exact or approximate ground state. We mention Kitaev's model (and perturbations thereof) on a hexagonal lattice which has the toric code state - a WGS - as ground state.
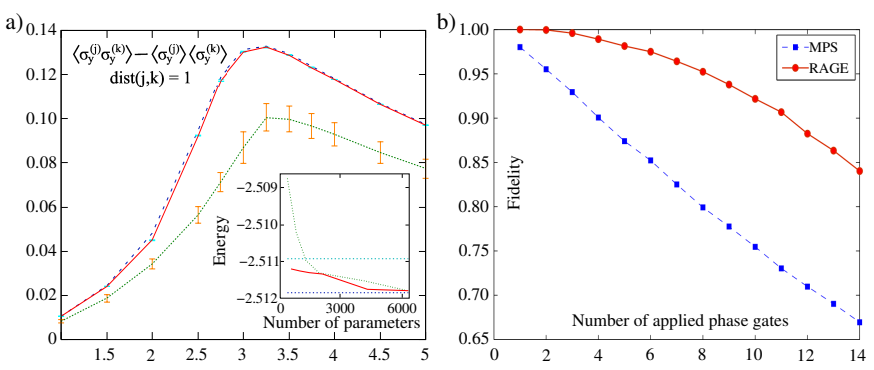

Figure 1: (a) 2D Ising model on a $4 \times 4$ periodic lattice. We compare the achievable accuracy with RAGE (red, solid) and MPS (green, dotted) with $D=4$ with exact results (blue, dashed). Two-point correlations as function of $B$ are shown. The inset depicts the energy for different total numbers of parameters and $B=2$ in comparison with the exact ground state (blue, dashed) as well as the first excited state (light blue, dashed). (b) Comparison of MPS (blue, dashed) and RAGE (red, solid) with $D=2$ for the simulation of a random quantum circuit [17] on $N=14$ qubits. Application of a random local phase gate followed by a random controlled-phase gate with random uniform phase in $[0,2 \pi)$ constitutes one block. For given $k$ we apply this block $k$ times to a randomly chosen initial MPS state. 500 such runs are determined, and in each the fidelity with the exact state is computed. The average over 500 realizations is then plotted.

Time evolution and simulation of quantum circuits. - We have also considered time evolution, more specifically the evolution of a quantum state in a quantum circuit. Here, sequences of elementary gates are applied, e.g., two-qubit phase gates and arbitrary single-qubit rotations. This method can be 
easily adapted to Hamiltonian (real or imaginary) time evolution. We now show how to efficiently obtain an optimal approximation of the resulting state after the application of an elementary gate. It turns out to be useful to restrict the variational family by setting $V_{j}^{(j)}=\mathbb{1}$, although an extension to arbitrary $V$ is possible. For phase gates, this update is particularly simple, as only a change in the adjacency matrix $\Phi$ is required. It is part of the strength of the scheme that phase gates between arbitrary constituents are already included in the variational set. The update of a local unitary will require some more attention:

Consider an initial state vector $|\psi(A, \Phi, \mathbb{1})\rangle$, to which a single qubit unitary operation $U$ is applied - acting, e.g., on the first qubit. The goal is now to find the best approximation $\left|\psi\left(A^{\prime}, \Phi^{\prime}, \mathbb{1}\right)\right\rangle$ which maximizes

$$
O:=\frac{\left|\left\langle\psi\left(A^{\prime}, \Phi^{\prime}, \mathbb{1}\right)\left|U_{1}\right| \psi(A, \Phi, \mathbb{1})\right\rangle\right|^{2}}{\left\langle\psi\left(A^{\prime}, \Phi^{\prime}, \mathbb{1}\right) \mid \psi\left(A^{\prime}, \Phi^{\prime}, \mathbb{1}\right)\right\rangle} .
$$

It appears natural to vary only phases that directly affect qubit 1, i.e., $\Phi_{j, k}^{\prime}=\Phi_{j, k}$ if $j \neq 1$. In this case, one can rewrite (5) in such a way that the optimal MPS part $A^{\prime}$ can be obtained analytically by solving a set of linear equations, while the optimization of a single phase $\Phi_{1, k}^{\prime}$ leads to a simple quadratic form. In practice, an alternating sweeping of both kinds of local variational methods is required. We have tested this method for a random quantum circuit (see Fig.1 (b)) and compared the achievable accuracy with MPS. Again, we obtain an improvement due to the WGS. Extensions. - A similar construction as illustrated for MPS also works for unifying WGS with other underlying tensor network descriptions. Similarly, one can use arbitrary clifford circuits instead of the WGS and can still efficiently contract. More precisely, whenever an exact or approximate evaluation of expectation values of arbitrary product observables (i.e., tensor products of local operators) for a state described by a tensor network is possible, then local observables (i.e., observables with a small support) can be efficiently computed for the unified family of such tensor network states and WGS (or clifford circuits), following an approach similar as in Eq. (3). While this certainly restricts the set of computable quantities (e.g., string-order parameters can no longer be evaluated), it still suffices to compute expectation values of all local Hamiltonians and hence one obtains a variational method for a ground-state approximation or simulation of quantum circuits.

Conclusions. - To summarize, we have introduced a new variational class of states to describe quantum many-body systems. These states have a number of desirable properties. Correlation functions can be computed efficiently, systematic improvements of the approximation within the class are possible and the states carry long-range correlations and violate entanglement area laws, as being encountered in critical systems or in quenched quantum systems undergoing time evolution. We have applied the RAGE ansatz to condensed matter and quantum computation problems, where we find an improvement over MPS. From a fundamental perspective the key question is where exactly the boundaries for the efficient classical description of quantum systems might lie. In fact, intriguingly, the entanglement content of the state cannot be taken as an indicator for the "complexity of a state" [18]. Delineating this boundary will reveal more about the structure of quantum mechanics from a complexity point of view and holds the potential for new improved algorithms and methods for the description of quantum systems.

Acknowledgements. - We thank S. Anders, T.J. Osborne and C.M. Dawson for illuminating discussions and the FWF, the EU (QAP, OLAQUI, SCALA), the EPSRC QIP-IRC, the Royal Society, Microsoft Research, and a EURYI for support.

[1] S.R. White, Phys. Rev. Lett. 69, 2863 (1992); Phys. Rev. B 48, 10345 (1993).

[2] U. Schollwöck, Rev. Mod. Phys. 77, 259 (2005).

[3] M. Fannes, D. Nachtergaele, and R.F. Werner, Commun. Math. Phys. 144, 443 (1992).

[4] S. Rommer and S. Ostlund, Phys. Rev. B 55, 2164 (1997); Phys. Rev. Lett. 75, 3537 (1995).

[5] G. Vidal, Phys. Rev. Lett. 91, 147902 (2003); G. Vidal, Phys. Rev. Lett. 93, 040502 (2004).

[6] F. Verstraete, D. Porras and J.I. Cirac, Phys. Rev. Lett. 93, 227205 (2004).

[7] K. Audenaert, J. Eisert, M.B. Plenio, and R.F. Werner, Phys. Rev. A 66, 042327 (2002); I. Peschel, J. Stat. Mech.: Th. Exp. P12005 (2004); A.R. Its, B.-Q. Jin, and V.E. Korepin, J. Phys. A 38, 2975 (2005); M.B. Plenio, J. Eisert, J. Dreissig, and M. Cramer, Phys. Rev. Lett. 94, 060503 (2005); M.M. Wolf, F. Verstraete, M.B. Hastings, and J.I. Cirac, arXiv:0704.3906, M.B. Hastings, arXiv:0705.2024.

[8] F. Verstraete, J.I. Cirac, Phys. Rev. B 73, 094423 (2006).

[9] P. Calabrese and J. Cardy, J. Stat. Mech. 0504, P010 (2005); J. Eisert and T.J. Osborne, Phys. Rev. Lett. 97, 150404 (2006); S. Bravyi, M.B. Hastings, and F. Verstraete, ibid. 97, 050401 (2006).

[10] G. Vidal, Phys. Rev. Lett. 99, 220405 (2007) arXiv:quant-ph/0610099. M. Stewart-Siu and M. Weinstein, arXiv:cond-mat/0608042; M. Rizzi, S. Montangero, arXiv:0706.0868

[11] C.M. Dawson, J. Eisert, and T.J. Osborne, arXiv:0705.3456

[12] F. Verstraete and J.I. Cirac, arXiv:cond-mat/0407066, V. Murg, F. Verstraete, and J.I. Cirac, Phys. Rev. A 75, 033605 (2007).

[13] W. Dür et al., Phys. Rev. Lett. 94, 097203 (2005).

[14] J. Calsamiglia et al., Phys. Rev. Lett. 95, 180502 (2005).

[15] S. Anders, M.B. Plenio, W. Dür, F. Verstraete, and H.J. Briegel, Phys. Rev. Lett. 97, 107206 (2006).

[16] S. Anders, H.J. Briegel, and W. Dür, New J. Phys. 9, 361 (2007).

[17] Y. Most, Y. Shimoni, and O. Biham, Phys. Rev. A 76, 022328 (2007).

[18] C. Mora and H.J. Briegel, Phys. Rev. Lett. 95, 200503 (2005); F. Benatti et al., Commun. Math. Phys. 265, 437 (2006).

[19] In our notation the the brackets in the upper position denote the support of an operator. 